\title{
Escolha de via de parto em uma maternidade de referência em Teresina-PI
}

\author{
Choice of delivery type in a reference maternity hospital in Teresina-PI \\ Elección del tipo de parto en maternidad de referencia en Teresina-PI
}

Laíme Ariádne Morena de Araújo ${ }^{1 *}$, José Francisco Ribeiro², Juliete de Jesus do Nascimento², Thamirys de Carvalho Mota $^{2}$, Elane Magalhães Oliveira² ${ }^{2}$ Thais da Silva Figueiredo ${ }^{3}$, Tassio Breno de Sousa Lopes Lavor ${ }^{1}$, Emanuele Cristina de Sousa Silva ${ }^{1}$.

\section{RESUMO}

Objetivo: Expectativas das primigestas na escolha da via de parto em uma maternidade pública de referência em Teresina. Metodologia: Trata-se de uma pesquisa estudo de campo do tipo exploratóriodescritivo de abordagem qualitativa, de forma que alcance os objetivos propostos e resultados esperados por meio das informações relevantes coletadas sobre o problema exposto. Resultados: Foram entrevistados vinte primigestas, sendo todas adultas, de idade entre 18 a 33 anos, contestando as escolhas das mulheres com a realidade do Estado, concluíram que os altos índices de partos cirúrgicos estão mais relacionados às escolhas feitas pelos profissionais, do que à vontade dos casais que vivenciam esse processo. Observamos a grande necessidade de um bom pré-natal, puérperas bem informadas sobre o seu ciclo gravídico. Conclusão: Ressaltando a fragilidade quando se trata da humanização e atenção no prénatal, uso da influência, tecnologias e intervenções desnecessárias e sobretudo, e pelo discurso biomédico e pela noção erronia, de que o parto cirúrgico seria o desfecho adequado para o nascimento.

Palavras-Chave: Parto normal e cesariana, Intervenções no parto, Pré-natal.

\begin{abstract}
Objective: Expectations of the primigravidae in choosing the type of delivery in a reference public maternity hospital in Teresina. Methodology: This is an exploratory-descriptive type of qualitative research, so that the objectives are proposed and the results obtained through the information are collected on the exposed problem. Results: Twenty primigrates, all adults, aged between 18 and 33 years old were interviewed, challenging women's choices with the reality of the State, and concluded that the high rates of surgical deliveries are more related to the choices made by professionals, than desire of the couples living this process. We noticed the great need for a good prenatal, well-informed mothers about the gestation period. Conclusion: Emphasis on fragility when it comes to humanization and attention in prenatal care, use of influence, unnecessary technologies and interventions and above all, and by the biomedical discourse and the notion of erronia, that the surgical delivery would be the appropriate outcome for the birth.
\end{abstract}

Keywords: Normal delivery and cesarean delivery, Interventions at birth, Prenatal.

\section{RESUMEN}

Objetivo: Expectativas de las primigestas en la elección de la vía de parto en una maternidad pública de referencia en Teresina. Metodología: Se trata de un estudio de campo del tipo exploratorio-descriptivo de abordaje cualitativo, de forma que alcance los objetivos y resultados esperados por medio de informaciones relevantes y relevantes sobre el problema. Resultados: Fueron entrevistadas veinte primigestas, siendo todas adultas, de edad entre 18 a 33 años, contestando las elecciones de las mujeres con la realidad del

\footnotetext{
${ }^{1}$ Universidade Estadual do Piauí (UESPI), Teresina-PI. *E-mail: ariad011@hotmail.com

2 Universidade Federal do Piauí (UFPI), Teresina-PI.

${ }^{3}$ Instituto Camilo Filho (ICF), Teresina-PI
} 
Estado, concluyeron que los altos índices de partos quirúrgicos están más relacionados a las elecciones hechas por los profesionales, la voluntad de las parejas que experimentan ese proceso. Observamos la gran necesidad de un buen prenatal, puérperas bien informadas sobre su ciclo gravídico. Conclusión: Resaltando la fragilidad cuando se trata de la humanización y atención en el prenatal, uso de la influencia, tecnologías e intervenciones innecesarias y sobre todo, y por el discurso biomédico y por la noción erronia, de que el parto quirúrgico sería el desenlace adecuado para el nacimiento.

Palabras-Clave: Parto cesárea normal, Intervenciones en el parto, Prenatal.

\section{INTRODUÇÃO}

Os cuidados prestados à mulher durante a maternidade sofreram uma grande mudança ao longo do tempo, principalmente a partir da segunda metade do século $\mathrm{XX}$, quando o parto passou a ser um evento hospitalar e cirúrgico. Este processo foi fundamental para o desenvolvimento do saber médico, culminando com o estabelecimento da medicalização do corpo feminino (NAGAHAMA e SANTIAGO, 2005).

No início da gestação, vários fatores influenciam na escolha sobre a via de parto desejada, dentre eles destacam-se os seguintes: o nível socioeconômico da gestante, seu contexto sociocultural, experiências reprodutivas anteriores e informações sobre os tipos de parto. Ao longo da gestação, informações recebidas, intercorrências clínico-obstétricas, influências familiares e do próprio médico no pré-natal poderiam modificar a escolha do tipo de parto, que pode ser feita pela mulher, pelo médico ou por ambos. No momento do parto, fatores relacionados à sua evolução e o próprio tipo de assistência oferecida poderiam alterar a escolha final da via de parto (DIAS, 2007).

A organização da assistência obstétrica restringe a possibilidade de escolha da mulher sobre o tipo de parto. O Estado financia a assistência que será ofertada nos serviços públicos ou privados contratados, os chamados serviços mistos. No Sistema único de saúde (SUS), as mulheres são geralmente acompanhadas por diferentes profissionais durante o pré-natal e assistência ao parto, com equipes nas maternidades atuando em regime de plantão (BRASIL, 2000).

Diante disso, é importante ter um conhecimento mais cientificado e relevante sobre quais são os reais fatores determinantes que influenciam as primigestas a escolherem a via de parto na nossa região, através do conhecimento sobre suas preferências, com o foco inicial na avaliação, visando as altas taxas de cesarianas desnecessárias ocorridas nos últimos anos, o que permite a obtenção de dados relevantes com os resultados obtidos, possibilitando orientações e ações educativas, com o intuito de minimizar o número de intervenções cirúrgicas desnecessárias.

\section{MÉTODOS}

Trata-se de uma pesquisa de estudo de campo do tipo exploratório-descritivo de abordagem qualitativa, de forma que alcance os objetivos propostos e resultados esperados por meio das informações relevantes coletadas sobre o problema exposto.

Foram incluídas na investigação 20 mulheres primigestas maiores de 18 anos e que estejam no puerpério mediato em uma maternidade pública de Teresina, durantes os meses da coleta. Para Araújo e Reis (2012) o puerpério mediato segue da $2^{a}$ hora ao $10^{\circ}$ dia pós-parto, cujas puérperas enfrentam maior risco de infecções genitais e extragenitais. Estas mulheres aceitaram voluntariamente participar da pesquisa mediante a leitura do Termo de Consentimento Livre e Esclarecido (TCLE). As entrevistas se encerraram quando ocorreu a saturação das falas, ou seja, uma repetição das respostas, portanto a saturação das falas representa $100 \%$ das puérperas (20) a serem entrevistadas. Para garantia do anonimato, os nomes originais das participantes da entrevista foram substituídos por depoentes.

Após a autorização da instituição envolvida na pesquisa e aprovação pela Comissão de Ética em Pesquisa da Universidade Estadual do Piauí (UESPI/FACIME) foram aplicados os roteiros de entrevistas. A 
princípio, foi realizado um convite individual às puérperas primigestas para participarem desta investigação de forma voluntária e, após o aceite, a entrevista foi realizada individualmente na unidade. Foi realizada previamente a Pré-Testagem do instrumento para sua validação.

A técnica utilizada neste estudo foi a entrevista, que de acordo com Gil (2011), é uma técnica que envolve duas pessoas numa situação "face a face", em que uma delas formula questões e a outra responde, não sendo dirigidas a uma categoria específica de pessoas, mas às pessoas que se relacionam com o fenômeno a ser pesquisado.

O instrumento para coleta de dados foi elaborado pelos pesquisadores, consistindo de um roteiro de entrevista semi-estruturada (APÊNDICE A), que para Marconi e Lakatos (2010), o entrevistador tem liberdade para desenvolver cada situação em qualquer direção que considere adequada. É uma forma de poder explorar mais amplamente uma questão, podendo ser respondida dentro de uma conversação informal. A entrevista foi gravada em MP3 com a permissão das entrevistadas, conforme suas assinaturas no Termo de Consentimento Livre e Esclarecido (TCLE), contido no ANEXO C, cujas respostas foram transcritas pelos próprios pesquisadores.

Os dados foram analisados logo após o parecer favorável do Comitê de Ética e Pesquisa (CEP) da Universidade Estadual do Piauí, sob o Certificado de Apresentação para Apreciação Ética (CAAE) no 37802314.7.0000.5209 (ANEXO A), e da Comissão de Ética e Pesquisa da Maternidade de referência (ANEXO B) e da comissão, por meio da análise temática, uma modalidade da técnica de análise de conteúdo. Para Minayo (2011), esta modalidade de análise origina-se de uma leitura de primeiro plano das falas, depoimentos e documentos, a fim de atingir um nível mais profundo, ultrapassando os sentidos manifestos do material, em que, operacionalmente, a análise temática desdobra-se nas seguintes etapas: pré-análise, exploração do material e tratamento dos resultados obtidos e interpretados. O referencial temático é considerado como principal fonte de informações e fundamentação para a elaboração das categorias e análise, pormenorizado nos depoimentos.

\section{RESULTADOS E DISCUSSÃO}

Foram entrevistadas vinte primigestas, todas adultas com faixa etária entre 18 e 33 anos, estando assim distribuídas de 18 a 25 anos com quatorze mulheres; entre 26 e 33 anos foram encontradas seis, em que a maioria delas era casada ou estavam em uma união estável; onze delas eram provenientes do interior do estado do Piauí, nove da capital Teresina e uma do interior do Maranhão.

Dezesseis puérperas referiram ter cursado até o ensino médio, sendo que, destas, apenas nove completaram este ciclo; quatro mulheres relataram cursar o ensino superior, e apenas duas concluíram o mesmo.

Em relação a exercer alguma atividade empregatícia, onze mulheres não trabalhavam fora de casa, sete delas trabalhavam no lar e quatro eram estudantes. Logo, a menor parte delas apresentou algum tipo de renda, de forma a garantir ou complementar o orçamento da família. Das mulheres entrevistadas, grande parte delas referiram que sua renda familiar era entre um e dois salários mínimos, três delas não têm renda fixa e contam com a ajuda do Programa Bolsa Família como a única renda para sustento, e uma delas referiu ter a renda de quatro a cinco salários mínimos.

O processo de parir vem sofrendo influência direta da cultura hospitalocêntrica. A reorganização de sua atenção, especialmente a partir do século XIX, construiu uma condição patológica a um evento que, até então, caracterizava-se como biológico e social (DINIZ, 2005)

As gestantes e seus acompanhantes, em sua grande maioria, declararam preferência pelo parto vaginal, contradizendo os números de partos cirúrgicos nacionais e confirmando os achados de outras pesquisas, em que a absoluta maioria das mulheres brasileiras aponta a via vaginal como melhor opção. Ao fazerem 
essa escolha, os sujeitos resgataram o parto como fenômeno fisiológico (FAÚNDES et al., 2004; DINIZ, 2005).

Contestando às escolhas das mulheres, com a realidade do Estado, observou-se que os altos índices de partos cirúrgicos estão mais relacionados às escolhas feitas pelos profissionais do que com a vontade dos casais que vivenciam esse processo, como o que pode ser constatado pelas afirmações a seguir:

[...] Tive cesariana, queria ter tido normal e menos dias de repouso e mais rápida a recuperação (Gestante 02).

[...] Tive cesariana, eu não queria, queria normal mesmo mais ai tive complicações e fiz cesariana, queria normal porque era mais rápido e eu podia voltar logo e trabalhar (Gestante 04).

[...] Tive cesariana, queria ter tido normal e mais rápida a recuperação e é menos sofrimento pra o meu bebe (Gestante 14).

Mesmo quando não existe risco materno ou fetal, as mulheres com melhores condições econômicas frequentemente optam por cesariana como forma de terminar a gravidez, tendo sido associada ao que seria um atendimento de qualidade. (BEHAGUE; VICTORA; BARROS, 2002; BARROS et al., 2011).

Embora outros estudos no Brasil apontem o parto cesariano como forma de alivio da dor e oportunidade para laqueadura tubária, o fator mais relacionado ao desfecho da cesárea eletiva foi a superestimação do risco em torno do parto normal. Esse achado também pode ajudar a refletir sobre a mudança de preferência ao longo do pré-natal, vinculada fortemente à interpretação do "parto normal arriscado" (BARBOSA et al., 2003; FIGUEIREDO et al., 2010), presente nos diálogos entre alguns médicos e as mulheres desta pesquisa:

[...] Tive cesariana, queria ter tido cesariana mesmo, é menos dor e sofrimento da hora. (Gestante 07)

Discutir as "preferências" reprodutivas das mulheres como uma questão de direitos exige o compromisso de ir mais além do que a própria opinião ou crença expressa, como proposto nos estudos encontrados. Assim, desafia-se o que é geralmente normatizado pela cultura e utilizado de modo nivelado para restrição à autonomia das mulheres.

Como sugere Martin (2006), a atuação e a autonomia das mulheres podem ou não estar relacionadas a um parto mais ou menos tecnologizado e centrado na figura médica, embora muitas vezes elas próprias pareçam não ter consciência das práticas opressoras presentes na vivência do parto e nascimento de seus filhos. Ao mesmo tempo, outras mulheres sentem que perderam ou foram impedidas de vivenciar suas escolhas e experiências com respeito.

A absorção do discurso médico-científico e a inferioridade imposta às mulheres em relação ao saber médico institucionalizado (CHACHAM, 2006) podem fazer com que muitas vezes elas se sintam incapazes de decidir o que é melhor para si e para o seu filho, delegando ao médico essa decisão. Tal exposto pode ser ratificado na seguinte fala de Gestante 16 , onde relatou:

\section{[...] Tive cesariana, não tinha preferência, o que fosse pra mim tava bom. (Gestante 16)}

Ao mesmo tempo, afirmar que todas as mulheres estão exercendo seus direitos reprodutivos pode acarretar distorções sobre as diversas realidades envolvidas, como aquelas relacionadas à própria concepção médica a respeito de seus corpos, o que as impossibilita de compreender e exercer concepções positivas de suas capacidades reprodutivas e nascimento de seus filhos, as escolhas e preferências baseadas no medo, de fato, não parecem permitir a autonomia sobre as decisões das mulheres. Muitas vezes, hipóteses, preferências e escolhas são construídas sob pressões familiares e das próprias normatizações provenientes da sociedade (CORRÊA e PETCHESKY, 1996)

Cada mulher atribui um significado às experiências vividas e essas experiências serão utilizadas em outras vivências (LOPES et al., 2008). Cabe, então, ao enfermeiro amenizar, no caso de mulheres 
socializadas em um meio culturalmente medicalizado, o embate com a abordagem humanizada. Essa mediação pode acontecer através das ações educativas em saúde que não só respeitem a individualidade, mas permitam a tomada de decisão pelo ser cuidado. Deste modo, a educação em saúde pode atuar como um potencializador do cuidado do enfermeiro, pois é capaz de gerar mudanças, o aprender mútuo e a construção de relações humanas simétricas (SANTOS; PENNA, 2009).

Foi observada a necessidade de um bom pré-natal, em que as puérperas pudessem ser bem informadas sobre o seu ciclo gravídico, bem como todos os sinais necessários serem identificados para que todas as condutas a serem realizadas durante a gravidez e na hora do parto, práticas de conforto da dor e manobras que ajudem na hora de parir, pudessem ser previamente detectadas, evitando assim intervenções desnecessárias.

[...] Tive orientações quando eu fui no posto com a enfermeira, me disse como seria o parto e disse pra eu me alimentar bem, com coisas leves e saudáveis e também pra observar o meu bebe, se ele tava mexendo, a conversar com ele (Gestante 18).

[...] A enfermeira me explicou cada um deles no pré-natal, me ensinou massagem e respiração pra hora do parto, disse que eu tinha direito de escolher como eu queria, ai eu escolhi o normal eu não tive uma influência, só achei que a dor só era na hora e depois não tinha mais, melhor que fazer um corte (Gestante 08).

Ainda que não seja uma constatação das puérperas, é possível inferir, a partir de seus depoimentos que, na dinâmica das consultas no pré-natal, observou-se que as mesmas não foram devidamente informadas tanto na rede suplementar, como na atenção básica. Havendo uma necessidade de produção de espaços mais privilegiados de escuta na relação profissional-usuário, essa problemática também sinaliza a urgência de investimentos no componente relacional do cuidado (MINAYO, 2007).

Vale ressaltar, mais uma vez, que é necessária uma conduta informativa no pré-natal. Foi observada uma grande deficiência de informação paras as puérperas, práticas de educação e saúde, e palestras educativas. A grande maioria das entrevistadas teve algum tipo de falta de informação, seja ela com relação às condutas de cada tipo de parto, ou em relação às dúvidas frequentes, durante a gestação.

[...] A enfermeira falou do parto normal, como era e que era melhor, minha família também falou que era melhor, disse que se recuperava rápido e eu poderia voltar a trabalhar mais cedo também sem precisar de repouso muito grande (Gestante 10).

[...] Tive orientações do parto normal, falo como era as dores e pra eu ir pra o hospital quanto tivesse essas dores ou se eu tivesse sangramento (Gestante 17).

[...] Tive orientações no pré-natal com a enfermeira, ela me disse como era o parto normal como eu sabia que tava perto de pari e como era a dor (Gestante 20).

Supondo que a implementação da Estratégia Saúde da Família (ESF), que prioriza ações de promoção à saúde das famílias, com destaque na atenção básica, Portanto, a reestruturação do modelo assistencial no país e o fortalecimento da atenção básica, via ESF, priorizam, entre outras, ações promocionais específicas ao período gravídico-puerperal (BRASIL, 2006).

Atenção ao pré-natal e ao nascimento na ESF, não disponibilizaram adequadamente práticas educativas, enfatizando assim o entendimento de que a atenção ao pré-natal tende a permanecer medicalizada e intervencionista, fazendo com que as puérperas fiquem despreparadas quanto ao seu estado gravídico desvalorizando a potencialidade do componente educativo como elemento qualificador da atenção e promotor de saúde.

Este conceito pode ser confirmado através das falas das entrevistadas:

[...] Não tive orientações, fiz o pré-natal mais não me explicaram nenhum dos dois (Gestante $11)$. 


\section{[...] Eu não tive orientações e não fiz pré-natal (Gestante 07).}

Embora a avaliação da qualidade do pré-natal tenha sido por meio de critérios que julgam o acesso, também deve ser considerado não somente o número de consultas ou a idade gestacional de início do acompanhamento, mas também a adequação do conteúdo da assistência prestada. Estudos demonstram que a avaliação do processo da assistência pré-natal contribui para a melhoria da qualidade dos serviços, bem como é fundamental para a redução dos índices de mortalidade materna e perinatal (KOFFMAN e BONADIO, 2005).

Concordando com os tais achados, uma pesquisa transversal de Domingues et al. (2004) com 246 mulheres, usuárias de uma maternidade pública do Rio de Janeiro, também elucida a questão do descaso com a informação disponibilizada às mulheres. Quando perguntadas sobre o quanto se sentiam informadas em relação ao que acontecia com elas e o bebê durante o parto, $23 \%$ declararam estarem completamente informadas e $43 \%$ consideraram-se não informadas. Menos da metade (44\%) referiu ter recebido informação pelo profissional que a acompanhou no pré-natal. Quando questionadas sobre as informações fornecidas pelos profissionais da instituição durante o trabalho de parto, apenas $60 \%$ sentiram-se informadas em relação à evolução do processo.

\section{CONCLUSÃO}

Frente aos achados do presente estudo, conclui-se que a maioria das puérperas são mulheres entre 18 e 25 anos, casadas, residentes no interior do estado do Piauí, com escolaridade satisfatória, e que não contribuem com a renda mensal da família, sendo que tal renda varia entre um a dois salários mínimos. Este estudo buscou entender, pelas perspectivas das próprias mulheres, o sentimento por traz da decisão da via de parto, compreendeu-se a importância de um pré-natal fidedigno, com informações precisas e esclarecedoras às beneficiadas pelo serviço de saúde a elas prestado. Foi observada também a importância de atividades complementares para as gestantes, com a finalidade de melhor fixação das informações adquiridas no pré-natal, apesar de reconhecer que é pouco viável que as participantes deste estudo recordem de todas as orientações recebidas nesse atendimento, bem como o interesse em conhecer o teor das informações disponibilizadas e o aprendizado adquirido por elas durante as consultas. Todas essas informações serão refletidas nas vivências futuras.

Os resultados deste estudo não se configuram em avaliação do atendimento ao pré-natal propriamente dito, ao mesmo passo em que não foge inteiramente à finalidade desta abordagem. O pré-natal é um reflexo imediato na escolha da mulher, mas o que observamos nos discursos aqui apresentados, sob a perspectiva das puérperas usuárias dos serviços de atenção pré-natal aponta à vulnerabilidade das mesmas, de modo a contribuir para que gestores, profissionais, docentes e estudantes da área da saúde, repensem as ações endereçadas ao período gravídico-puerperal para acrescentar na resolutividade a esta problemática. Foi um longo caminho de amadurecimento, que me permitiu compreender a importância da incorporação do diálogo sobre os direitos humanos das mulheres para a interpretação e crítica aos tortuosos caminhos das escolhas na experiência do parto no Brasil.

\section{REFERÊNCIAS}

1. ANDREUCCI CB, CECATTI JG. Desempenho de indicadores de processo do Programa de Humanização do Prénatal e Nascimento no Brasil: uma revisão sistemática. Cad Saude Publica, 2011; 27(6).

2. Parto cesáreo: quem o deseja? Em quais circunstâncias? Cadernos de Saúde Pública, Rio de Janeiro 2003. Disponível em: http://www.scielo.br/pdf/csp/v19n6/a06v19n6.pdf. Acesso em: 09 jun. 2015.

3. BARROS AJD, SANTOS IS, MATIJASEVICH $A$ et al. Patterns of deliveries in a Brazilian birth cohort: almost universal cesarean sections for the better-off. Rev Saúde Pública, 2011;45.

4. BEHAGUE DP, VICTORA CG, BARROS FC et al. Consumer demand for caesarean sections in Brazil: informed decision making, patient choice, or social inequality? A population based birth cohort study linking ethnographic and epidemiological methods. BMJ, 2002; 5.

5. Brasil. Agência Nacional De Saúde Suplementar. O modelo de atenção obstétrica no setor de saúde suplementar no Brasil: cenários e perspectivas. Rio de Janeiro 2008 . Disponível em http://www.ans.gov.br/portal/upload/noticias/clipping/livro parto web.pdf. Acesso em 30 set 2014. 
6. CORREAA S, PETCHESKY R. Direitos sexuais e reprodutivos: uma perspectiva feminista. Revista de Saúde Coletiva, $1996 ; 6$, n. $1 / 2$.

7. CYR R M. Myth of the ideal cesarean section rate. Commentary and historic perspective. Am J Obstet Gynecol, 2006; 6.

8. D'ORSI E, BRÜGGEMANN OM, DINIZ CSG et al. Desigualdades sociais e satisfação das mulheres com o atendimento ao parto no Brasil: estudo nacional de base hospitalar. Cad Saúde Publica 2014.

9. DECLERCQ EF, MENACKER F, MACDORMAN M et al. Maternal risk profiles and primary cesarean rate in the United States. Am J Public Health,2006; 96.

10. DIAS MAB. Trajetória das mulheres na definição pelo parto cesáreo: estudo de caso em duas unidades do sistema de saúde suplementar do estado do Rio de Janeiro. Ciênc. saúde coletiva, 2008;(13); 5.

11. DIAS MAB, DOMINGUES RMSM. Desafios na implantação de uma política de humanização da assistência hospitalar ao parto. Ciên \& Saúde Coletiva, 2005; (10); 3.

12. DIAS MAB, DOMINGUES RMSM. Trajetória das mulheres na definição pelo parto cesáreo: estudo de caso em duas unidades do sistema de saúde suplementar do estado do Rio de Janeiro. Associação Brasileira de Saúde Coletiva. Rio de Janeiro, 2007 (13); 5.

13. DINIZ CSG. Humanização da assistência ao parto no Brasil: os muitos sentidos de um movimento. Ciênc Saúde Colet , 2005, (10); 3.

14. DINIZ SG. Gênero, saúde materna e o paradoxo perinatal. Rev Bras Crescimento Desenvolv Hum, 2009,(19); 2.

15. DOMINGUES RMS, SANTOS EM, LEAL MC et al. Aspectos da satisfação das mulheres com assistência ao parto: contribuição para o debate. Cadernos de Saúde Pública, Rio de Janeiro, Suplemento 1,2004; 20.

16. ENKIN M, KEIRSE MJNC, NEILSON J et al. A Guide to Effective Care in Pregnancy and Childbirth. England: Oxford University Press; 2000.

17. FABRI RH, LIMA E, SILVA HS et al. Estudo comparativo de cesariana entre um hospital público universitário e um hospital privado. Rev Bras Saúde Matern Infant, 2002, 2.

18. FAUNDES A, PÁDUA KS, OSIS MJD et al. Opinião de mulheres e médicos brasileiros sobre a preferência pela via de parto. Rev Saúde Pública, 2004; 38, (4).

19. FIGUEIREDO NSV et al. Fatores culturais determinantes da escolha da via de parto por gestantes. HU Revista, $2010 ; 36$

20. HUMPSTONE OP. Cesarean section vs spontaneous delivery. Am J Obstet Gynecol, 1920; 1.

21. KOFFMAN MD, BONADIO IC. Avaliação da atenção pré-natal em uma instituição filantrópica da cidade de São Paulo. Rev. Bras. Saude Mater. Infant., 2005; 5,(1).

22. MARTIN E. A mulher no corpo: uma análise cultural da reprodução. Rio de Janeiro: Garamond, 2006.

23. MARTINS AL. Mortalidade materna de mulheres negras no Brasil. Cadernos Saúde Pública, Rio de Janeiro, 2006; $22(11)$.

24. MINISTÉRIO DA SAÚDE. 2001 Parto, Aborto e Puerpério Assistência Humanizada à Mulher. Brasilia DF. Disponível em: http://bvsms.saude.gov.br/bvs/publicacoes/cd04 13.pdf Acesso em 30 de set. 2014.

25. NAGAHAMA EEI, SANTIAGO SM. Práticas de atenção ao parto e os desafios para humanização do cuidado em dois hospitais vinculados ao Sistema Único de Saúde em município da Região Sul do Brasil. Cad Saúde Pública, 2008; 24 (8).

26. OLIVEIRA SMJV, MIQUILINI EC. Frequência e critérios para indicar a episiotomia. Rev Esc Enferm USP, $2005 ; 39$.

27. Organização Mundial da Saúde. Maternidade segura: atenção ao nascimento normal: guia prático. Genebra: Organização Mundial da Saúde; 1997.

28. POTTER JE, HOPKINS K, FAUNDES A et al. Women's autonomy and scheduled cesarean sections in Brazil: a cautionary tale. Birth, 2008; 35.

29. RATTNER D. Humanização na atenção a nascimentos e partos: ponderações sobre políticas pública. Interface comun saúde educ , 2009; 13 (1).

30. REIS AED, PATRÍCIO ZM. Aplicação das ações preconizadas pelo Ministério da Saúde para o parto humanizado em um hospital de Santa Catarina. Ciênc Saúde Coletiva, 2005; 10.

31. SANTOS JO, BOLANHO IC, MOTA JQC et al. Frequência de lesões ocorridas nos partos vaginais em uma instituição hospitalar. Esc Anna Nery Rer Enferm, 2008; 12 (4).

32. SCHENKER JG, CAIN JM. FIGO Committee Report. FIGO Committee for the Ethical Aspects of Human Reproduction and Woman's Health. International Federation of Gynecology and Obstetrics. In J Gynaecol Obstet. 1999.

33. Secretaria de Políticas de Saúde, Ministério da Saúde. Programa de Humanização do Pré-Natal e Nascimento. Brasília: Ministério da Saúde; 2000.

34. TESSER C. D.; Medicalização social (I): o excessivo sucesso do epistemicídio moderno na saúde. Interface Comunic, Saúde, Educ, Botucatu, SP, 2006; 10 (19).

35. WOLFF LR, WALDOW VR. Violência consentida: mulheres em trabalho de parto e parto. Saúde soc.,2008; 17 (3). 